\title{
Diversity and Composition of Tree Species under Semi-arid Uttar Pradesh, India
}

\author{
Sonam Rajput ${ }^{1 *}$ and P.K. Varshney ${ }^{2}$ \\ ${ }^{1}$ Department of Silviculture and Agroforestry, College of Forestry, Dr. YSP University, \\ Nauni, Solan, Himachal Pradesh, India \\ ${ }^{2}$ Department of forestry, school of life sciences, DBRA University, Agra, Utter Pradesh, India \\ *Corresponding author
}

\section{A B S T R A C T}

\begin{tabular}{|l|}
\hline Ke y w o r d s \\
Natural and \\
artificial \\
regeneration, \\
$\begin{array}{l}\text { Family wise, } \\
\text { Habitat wise, Tree } \\
\text { species }\end{array}$ \\
\hline Article Info \\
\hline $\begin{array}{l}\text { Accepted: } \\
\text { 10 May } 2019 \\
\text { Available Online: } \\
\text { 10 June } 2019\end{array}$ \\
\hline
\end{tabular}

\section{Keywords}

Natural and

Habitat wise, Tree

Article Info

Accepted:

Available Online

10 June 2019

\section{Introduction}

Regeneration of major canopy tree species has been studied in many forest ecosystems (Denslow, 1987; Yamamoto, 1996). Tropical forests revealed variation in patterns of regeneration both through differences in their constituent species and the environmental variables in which they grow (Denslow, 1987; Garwood, 1989; Whitmore 1996; Teketay 1997a; Kyereth et al., 1999). Such works have shown that studies on natural regeneration and seedling ecology can provide options to forest development through improvement in recruitment, establishment and growth of the desired seedlings. Also studies on tree seedlings density, their rate of mortality and damage help in the understanding of the status of species and natural regeneration (Augspurger, 1984; Hubbel and Foster, 1986).

The major component for the formation of forest communities is the woody species. The nature of forest communities largely depends 
on the ecological characteristics in sites, species diversity and regeneration status of species.

Micro environmental factors vary with seasonal changes which affect the growth stage i.e. seedling, sapling and young trees of the plant communities that maintain the population structure of any forest. Hence, it becomes an important issue to understand the tree diversity, population structure and regeneration status of forest communities for the maintenance of both natural and control forest.

The satisfactory natural regeneration behaviour of the forests largely depends on population structure characterized by the production and germination of seed, establishment of seedlings and saplings in the forest (Rao, 1988).

Complete absence of seedlings and saplings of tree species in a forest indicates poor regeneration, while presence of sufficient number of young individuals in a given species population indicates successful regeneration (Saxena and Singh, 1984). However, the presence of sufficient number of seedlings, saplings and young trees is greatly influenced by interaction of biotic and abiotic factors of the environment (Boring et al., 1981; Aksamit and Irving, 1984).

Tree population structure and its implication for their regeneration has been studied in different forest communities of India e.g. Garhwal (Baduni and Sharma, 2001; Bhandari, 2003), Himachal Pradesh (Sood and Bhatia, 1991), Western Himalayas (Pande et al., 2002), Western Ghats (Parthasarathy, 2001) and north eastern region (Yadava et al., 1991; Maram and Khan, 1998; Bhuyan et al., 2002, 2003).

An introduced, alien, exotic, non-indigenous, or non-native species, or simply an introduction, is a species living outside its native distributional range, which has arrived there by human activity, either deliberate or accidental. Non-native species can have various effects on the local ecosystem. Introduced species that have a negative effect on a local ecosystem are also known as invasive species.

Not all non-native species are considered invasive. Some have no negative effect and can, in fact, be beneficial as an alternative to pesticides in agriculture for example. In some instances the potential for being beneficial or detrimental in the long run remains unknown. A list of introduced species is given in a separate article (Carlton, 2002).

\section{Materials and Methods}

The study was carried out from Agra district of Uttar Pradesh, which is located $27.1767^{\circ} \mathrm{N}$ latitude and $78.0081^{\circ} \mathrm{E}$ longitude. This study was undertaken during different months of year 2014. Month viz. (April, May, June \& July 2014). The study had been done in different habitat wise (i.e. agricultural land, protected area, unprotected area \& road side) and family diversity wise.

The study was divided in to two parts that is artificial and natural regeneration. Five selected area of this study were Tajnature park, Shajahan garden, Paliwal park, company garden and Mau forest. The primary information such as local name, ecological condition of occurrence, status, growth, habitat condition etc. for each species, was collected. The nativity of the species was identified (samant et al., 1998).

Endemism of the species was identified based on distribution of the species (Dhar and Samant, 1993).

\section{Results and Discussion}




\section{Tree composition according there sites}

Site: 1 (Taj nature walk) - consist (natural regeneration), maximum area covered by road side plantation (36 percent) followed by protected area (29percent) and unprotected area (21percent) respectively while minimum (14percent) under agricultural land.

In artificial regeneration was carried out in following manner, maximum area (29\%) under protected condition followed by (percent each) in protected and road side area while minimum area (21percent) under agricultural land.

Site: 2 (Shajahan garden) - consist maximum area (28percent each) comes under unprotected and road side plantation followed by (22percent each) under protected area and agricultural land.

In artificial regeneration was carried out in following manner, maximum area (23percent) possessed unprotected area while (24.25percent each) area under agricultural land and road side plantation and but minimum area (20percent) under protected area.

Site: 3 (Paliwal park) - consist (natural regeneration), maximum area was covered by road side plantation and agricultural land (31percent) and followed by unprotected area (23percent) respectively while minimum (15percent) under protected area.

In artificial regeneration was carried out in following manner, maximum area (29percent) under protected area followed by (26percent) unprotected area and (23.35percent) agricultural land respectively while minimum area (22.05percent) road side plantation.
Site: 4 (Companygraden) - consist (natural regeneration), maximum area was covered by unprotected area (36percent) and followed by agricultural land (29percent) and protected area (21percent) respectively while minimum area (14percent) road side plantation.

In artificial regeneration was carried out in following manner, maximum area (36percent) under unprotected area condition followed by (28percent) road side plantation and (24percent) protected area respectively while minimum area (12percent) agricultural land.

Site: 5 (Mau forest) - consist (natural regeneration), maximum area was covered by equally (25percent each) agricultural land, protected area, unprotected area and road side plantation.

Tree composition family wise according there sites

Site: 1 (Taj nature park) -maximum tree species (23percent each) belong to moraceae and fabaceae which followed by (14percent) meliacea respectively but minimum percentage (8percent each) tree species which occurred under following families leguminose, bignoniaceae, rutaceae, and moringaceaein natural regeneration.

At site 1 maximum tree species (14 percent) belong to moraceae family followed by (11 percent) apocynaceae, (11 percent) meliaceae, (8 percent) lamiaceae, ( 8 percent) leguminose, (8 percent) fabaceae respectively while minimum percentage (14 percent each) occur under following families-annonaceae, rubiaceae, putanjaceae, sapotaceae, salvadoraceae, casuarinaceae, ulmaceae, combretacea, lythraceae, and cupressaceae in artificial regeneration (Table 1 and 2). 
Table.1 Diversity of natural \& artificial regenerated Tree species at different site

\begin{tabular}{|c|c|c|c|}
\hline \multicolumn{2}{|c|}{ Artificial regeneration } & \multicolumn{2}{|r|}{ Natural regeneration } \\
\hline Habitat & Plant species & Habitat & Plant species \\
\hline $\begin{array}{l}\text { Agricultural } \\
\text { land }\end{array}$ & $\begin{array}{l}\text { Aeglemarmelos }(\mathrm{L} .) \\
\text { Tamarindusindica }(\mathrm{L} .) \\
\text { Pongamiapinneta }(\mathrm{P} \text { glabra) } \\
\text { Neriumindicum }(\mathrm{Mill} .) \\
\text { Azadirachtaindica }(\mathrm{Sl}) \\
\text { Mimusopselengi }(\mathrm{L} .) \\
\text { Syziumcumini }(\mathrm{L} .) \\
\text { Alstoniascholaris }(\mathrm{L} . \mathrm{R} . B r .) \\
\text { Zizyphusmouritiana }(\text { Lam. }) \\
\text { Lowsonia alba }(\mathrm{L} .) \\
\text { Calistamoncancolates }(\mathrm{R} . B r .) \\
\text { Meliaazedarach }(B) \\
\text { Morus alba }(\mathrm{Qg}) \\
\text { Moringaolefiera }(\mathrm{Lam} .) \\
\text { Embilicaofficinalis }(\mathrm{L} .) \\
\text { Delbergiasisso }(\mathrm{P})\end{array}$ & $\begin{array}{l}\text { Agricultural } \\
\text { land }\end{array}$ & $\begin{array}{l}\text { Pongamiapinnata }(\mathrm{P} \text { glabra) } \\
\text { Meliaazedarach }(B) \\
\text { Ficusreligiosa }(\mathrm{L} .) \\
\text { Ricinuscommunis }(\mathrm{L} .) \\
\text { Embilicaofficinals }(\text { Gaerth }) \\
\text { Syziumcumini }(\mathrm{L} .) \\
\text { Aeglemarmelos }(\mathrm{L} .) \\
\text { Moringaolefera }(\mathrm{Lam} .) \\
\text { Zizyphusmouritiana }(\text { Lam. }) \\
\text { Neriumindicm }(\text { Mill. }) \\
\text { Meliaazedarach }(B) \\
\text { Moringaolefiera }(\mathrm{Lam} .) \\
\text { Azadirachtaindica }(\mathrm{Sl}) \\
\text { Mimusopselengi }(\mathrm{L} .)\end{array}$ \\
\hline Protected area & $\begin{array}{l}\text { Polyalthialongifolia(Som.) } \\
\text { Hiterophragmaadenophyllum } \\
\text { Dypsislutescens(H. wendl.) } \\
\text { Phonixsylvestris(1.) } \\
\text { Casurianaequisetifolia(Fs.) } \\
\text { Tetonagrandis(B) } \\
\text { Mitragynaparvifolia(Roxb.) } \\
\text { Putranjivaroxburghi(P.) } \\
\text { Terminaliabellirica(B) } \\
\text { Tecoma undulate(D.Don) } \\
\text { Thujacompacata } \\
\text { Neriumindicm (Mill.) } \\
\text { Thevetiapawiana(Pers.) } \\
\text { Meliaazedarach }(B) \\
\text { Ailanthus excelsa(P) } \\
\text { Alstoniascholaris(L.R.Br.) } \\
\text { Callistemon citrinus(R. Br.) } \\
\text { Moringaolefiera(Lam.) } \\
\text { Saracaindica }(\text { Sosaca) } \\
\text { Salvadoraoleoides(Dence.) } \\
\text { Albizzialebbeck(L.) } \\
\text { Parkinsoniaaculeata (L.) }\end{array}$ & $\begin{array}{l}\text { Protected } \\
\text { area }\end{array}$ & $\begin{array}{l}\text { Cassia siamea }(\mathrm{Qg}) \\
\text { Cassia fistula }(\mathrm{Qg}) \\
\text { Ailanthus exclesa }(\mathrm{P}) \\
\text { Ficusinfectoria }(\text { F.lacer }) \\
\text { Tecoma undulate }(\mathrm{D} . \mathrm{Don}) \\
\text { Meliaazedarach }(\mathrm{B}) \\
\text { Azadirachtaindica }(\mathrm{Sl}) \\
\text { Albizzialebbeck }(\mathrm{L} .) \\
\text { Neriumindicm }(\text { Mill. }) \\
\text { Ficusbengalensis }(\mathrm{L} .) \\
\text { Pongamiapinnata }(\mathrm{p} \text { glabra) } \\
\text { Saracaindica }(\text { Sosaca }) \\
\text { Capprisaphylla }(\text { Forssk.) } \\
\text { kigeliapinnata }(\mathrm{Jacq} .) \text { Dc. } \\
\text { Prosopisjuliflora(p. chinensis) }\end{array}$ \\
\hline
\end{tabular}




\begin{tabular}{|c|c|c|c|}
\hline & Cassia siamea $(\mathrm{Qg})$ & & \\
\hline $\begin{array}{l}\text { Unprotected } \\
\text { area }\end{array}$ & $\begin{array}{l}\text { Neriumindicum (Mill.) } \\
\text { Dalbergiasisso(P) } \\
\text { Moringaolefera(Lam.) } \\
\text { Alstoniascholaris(L.R.Br.) } \\
\text { Morus alba }(\mathrm{Qg}) \\
\text { Salvadoraoleoides(Dence.) } \\
\text { Hiterophragmaadenophyllum } \\
\text { Polyalthialongifolia(som.) } \\
\text { Thevetiapawiana(Pers.) } \\
\text { Mitrgyanaparvifolia(Roxb.) } \\
\text { Mimusopeselengi (L.) } \\
\text { Tecomaundulata(D.Don) } \\
\text { Dypsislutescens(H. wendl.) } \\
\text { Phonixsylvestris(1.) } \\
\text { Saracaindica(Sosaca) } \\
\text { Casurianaequisetifolia(Fs.) } \\
\text { Pongamiapinnata(p glabra) } \\
\text { Aeglemarmelos((L.) } \\
\text { Callistemon citrinus(R. Br.) } \\
\text { Thujacompacta(L.) }\end{array}$ & $\begin{array}{l}\text { Unprotected } \\
\text { area }\end{array}$ & $\begin{array}{l}\text { Holopteleaintegrfolia }(\mathrm{ROXB}) \\
\text { kigeliapinnata }(\mathrm{Jacq} .) \mathrm{Dc} \\
\text { Saracaindica }(\text { S osaca) } \\
\text { Capparisaphylla }(\text { Forssk.) } \\
\text { Thevitiaparwiana }(\text { Pers. } \\
\text { Meliaazedarach }(\text { B }) \\
\text { Cassia siemea }(\mathrm{Qg} .) \\
\text { Prosopisjuliflora }(\mathrm{p} . \text { chinensis) } \\
\text { Cassia fistula }(\mathrm{Pt} .) \\
\text { Azadirachtaindica }(\mathrm{Sl}) \\
\text { Pongamiapinnata }(\mathrm{p} \text { glabra) } \\
\text { Neriumindicm }(\text { Mill. }) \\
\text { Moringaolefiera }(\mathrm{Lam} .) \\
\text { Ficusbengalensis }(\mathrm{L} .) \\
\text { Ricinuscommunis }(\mathrm{L} .)\end{array}$ \\
\hline Road side & $\begin{array}{l}\text { Albizzialebbeck(Qg) } \\
\text { Thevetiaparwiana(Pers.) } \\
\text { Saracaindica(S osaca) } \\
\text { Hiteropheagmaadinofullam } \\
\text { Azadirachtaindica(Sl) } \\
\text { Dalbergiasisso(P) } \\
\text { Cassia siemea(Qg.) } \\
\text { Meliaazedarach }(\text { B) } \\
\text { Holpteleaintegrifolia(ROXB) } \\
\text { Phonixsylvestris(1.) } \\
\text { Alstoniascholaris(L.R.Br.) } \\
\text { Dypsislutescens(H. wendl.) } \\
\text { Ficusreligiosa(L.) } \\
\text { Ficusbengalensis(L.) } \\
\text { Casuarinaequisetifolia(Fs.) } \\
\text { Tecomaundulata(D.Don) } \\
\text { Cassia fistula (Pt.) } \\
\text { Ficusinfectoria(F.lacer) }\end{array}$ & Road side & $\begin{array}{l}\text { Prosopisjuliflora }(\mathrm{P} . \text { chinensis) } \\
\text { Thevitiaparwiana }(\mathrm{Pers} .) \\
\text { Ficusbengelensis }(\mathrm{L} .) \\
\text { Ficusreligiosa }(\mathrm{L} .) \\
\text { Azadirachtaindica }(\mathrm{Sl}) \\
\text { Meliaazedarach }(\mathrm{B}) \\
\text { Eucalyptus tertecornis }(\mathrm{Qg}) \\
\text { Acacia nilotica }(\mathrm{Pt} ., \text { Ar. }) \\
\text { Cassia siamea }(Q g) \\
\text { Ricinuscommunis }(\text { L. }) \\
\text { Albizzialebbeck }(\mathrm{L} .) \\
\text { Pongamiapinnata }(\mathrm{p} \text { glabra }) \\
\text { Holpteleaintegrifolia }(\mathrm{ROXB})\end{array}$ \\
\hline
\end{tabular}


Table.2 Diversity of natural \& artificial regenerated tree species at different site

\begin{tabular}{|c|c|c|c|}
\hline \multicolumn{2}{|c|}{ Artificial regeneration } & \multicolumn{2}{|c|}{ Natural regeneration } \\
\hline Family & & Family & Plant species \\
\hline Fabaceae & $\begin{array}{l}\text { Saracaindica }(\text { Sosaca) } \\
\text { Cassia fistula }(\mathrm{Pt} .) \\
\text { Prosopisjuliflora }(\mathrm{p} . \text { chinensis }) \\
\text { Albizzialebbeck }(\mathrm{L} .) \\
\text { Pongamiapinnata }(\mathrm{p} \text { glabra) } \\
\text { Parkinsoniaaculeata }(\text { L.) } \\
\text { Delbergiasisso }(\mathrm{P})\end{array}$ & Fabaceae & $\begin{array}{l}\text { Pongamiapinneta(p glabra) } \\
\text { Saracaindica(Sosaca) } \\
\text { Cassia fistula(Pt.) } \\
\text { Prosopisjuliflora(p. } \\
\text { chinensis) } \\
\text { kigeliapinnata(Jacq.)Dc }\end{array}$ \\
\hline Rutaceae & Aeglemarmelos $((\mathrm{L})$. & Rutaceae & Aeglemarmelos(L.) \\
\hline Lamiaceae & $\begin{array}{l}\text { Tectonagrandis }(\mathrm{B}) \\
\text { Hiterophragmaadenophyllum }\end{array}$ & Meliaceae & $\begin{array}{l}\text { Meliaazedarach }(B) \\
\text { Ailanthus excelsa }(\mathrm{p}) \\
\text { Azadirachtaindica }(\mathrm{Sl})\end{array}$ \\
\hline \multirow[t]{2}{*}{ Annonaceae } & Polyalthialongifolia(som.) & Leguminosae & Cassia siemea(Qg.) \\
\hline & $\begin{array}{l}\text { Azadirachtaindica }(\mathrm{Sl}) \\
\text { Ailanthus excels }(\mathrm{P}) \\
\text { Meliaazedarach }(B)\end{array}$ & Eubharbiaceae & Ricinuscommunis(L.) \\
\hline \multirow[t]{2}{*}{ Moraceae } & Ficusreligiosa(L.) & & Hiterophragmaadenophyllum \\
\hline & $\begin{array}{l}\text { Neriumindicm (Mill.) } \\
\text { Thevetiaparwiana(Pers.) } \\
\text { Alstoniascholaris(L.R.Br.) }\end{array}$ & Myrtaceae & Syzygiumcumini(L.) \\
\hline Arecaceae & $\begin{array}{l}\text { Dypsislutescens(H. wendl.) } \\
\text { Phonixsylvestris(1.) }\end{array}$ & Moraceae & $\begin{array}{l}\text { Ficusreligiosa }(\mathrm{L} .) \\
\text { Ficusbengalensis }(\mathrm{L} .) \\
\text { Ficusinfactoria }(\mathrm{F} . \text { lacer }) \\
\text { Morus alba }(\mathrm{Qg} .)\end{array}$ \\
\hline Leguminosae & $\begin{array}{l}\text { Cassia siamea }(\mathrm{Qg}) \\
\text { Tamarindusindica }(\mathrm{L} .)\end{array}$ & Apocynaceae & $\begin{array}{l}\text { Neriumindicm(Mill.) } \\
\text { Thevitiaparwiana(Pers.) }\end{array}$ \\
\hline Myrtaceae & Callistemon citrinus( $\mathrm{R} . \mathrm{Br})$. & Sapotaceae & Mimusopeselengi(L.) \\
\hline Ulmaceae & Holopteleaintegrifolia(ROXB) & Ulmaceae & Holpteleaintegrifolia(ROXB) \\
\hline Rhamnaceae & Zizyphusmouritiana(Lam.) & Moringaceae & Moringaolefiera(Lam.) \\
\hline Phyllanthoacea & Embilicaofficinalis(L.) & Bignoniaceae & Tecomaundulata(D.Don) \\
\hline Salvadoraceae & Salvadoraoleoides(Dence.) & & Zizyphusmouritiana(Lam.) \\
\hline Moringaceae & Moringaolefera(Lam.) & Capparaceae & Capprisaphylla(Forssk.) \\
\hline Casuarinaceae & Casuarinasequisetifolia(Fs.) & & \\
\hline Rubiaceae & Mitrgyanaparvifolia(Roxb.) & & \\
\hline Putranjaceae & Putranjivaroxburghii(P.) & & \\
\hline Capparaceae & Capprisaphylla(Forssk.) & & \\
\hline Bignoniaceae & Tecomaundulata(D.Don) & & \\
\hline Combretacea & Teminaliabellerica(Roxb.) & & \\
\hline Cupressaceae & Lowsoniaalba(L.) & & \\
\hline Lythraceae & Thujacompacta(L.) & & \\
\hline Sapotaceae & Mimusopeselengi (L.) & & \\
\hline
\end{tabular}


Site: 2 (Shajhan garden)- maximum tree species (21.25percent) belong to fabaceae which followed by (14percent each) meliaceae, apocynaceae and moraceae respectively but minimum percentage (7.25percent each) tree species which occurred under following families moraceae, putanjaceae, sapotaceae, rhamnaceae, ulmaceae and moringaceae in natural regeneration.

At site 2 maximum tree species (21percent) belong to fabaceae family followed by (11percent each) meliaceae, apocynaceae and moringaceae respectively while minimum percentage (4percent each) occur under following families - annonaceae, rhamnaceae, moringaceae, casuarinaceae, rutaceae, myrtaceae, sapotaceae, arecaceae, lamiaceae, ulmaceae and lythraceae in artificial regeneration.

Site: 3 (Paliwal park) - maximum tree species (44.50percent) belong to fabaceae which followed by (22.50percent) meliaceae, respectively but minimum percentage (11percent each) tree species which occurred under following families - moraceae, apocynaceae, and moringaceae in natural regeneration.

At site 3 maximum tree species (13percent each belong to meliaceae and apocynaceae which followed by (10.50percent) arecaceae and (7.50percent) fabaceae respectively but minimum percentage (4percent each) of tree species which occurred under following families - moraceae, phyllanthoceae, salvadoraceae, leguminose, ulmaceae, moringaceaecasuarinaceae, putranjaceae, myrtaceae, sapotaceae, annonaceae, lamiaceae, lythraceae and cupressaceae in artificial regeneration.

Site: 4 (Company garden) - maximum tree species (37percent) belong to fabaceae which followed by (15percent) meliaceae but minimum percentage (8percent each) apocynaceae, leguminose, eubharbiaceae, rhamnaceae and capressaceae in natural regeneration.

At site 4 maximum tree species (16percent each) belong to meliaceae and apocynaceae family followed by (11percent each) fabaceae, and arecaceae respectively while minimum percentage (5percent each) occur under following families - lamiaceae, myrtaceae, annonaceae, ulmaceae, rutaceae, arecaceae and lythraceae in artificial regeneration.

Site: 5 (Mau forest)-maximum tree species (43percent) belong to fabaceae but minimum percentage (14.25percent each) tree species which occurred under following families meliaceae, ulmaceae, leguminose and myrtaceae in natural regeneration.

At site 5 maximum tree species (49percent) apocynaceae but minimum percentage (17percent each) tree species occurred under following families -meliaceae, lamiaceae and rhamnaceae in artificial regeneration.

\section{Tree composition (Habitat wise)}

During survey period various habitat wise composition was noted in all sites as maximum tree species occurred (25.25percent each), protected area, unprotected area and agricultural land while minimum occur at (24.25percent) road side plantation in natural habitat.

In same manner, maximum (30percent) tree composition occurred in protected area, followed by (25percent) unprotected area and road side plantation (23percent) respectively while minimum (22percent) area under agricultural land, in artificial habitat. 


\section{Tree composition (Family wise)}

In natural regeneration, total 24 type of tree species which belong to 15 families were recorded at all sites in which maximum (20percent) of tree species belong to fabaceae which followed by (16percent) moraceae respectively.

Total 37 types of tree species which belong to 24 families were recorded, in artificial regenerated tree species in all sites. In which maximum number (17percent) of tree species belong to family fabaceae, followed by (7percent) meliaceae and apocynaceae respectively.

\section{Acknowledgement}

The author are highly thankfully to teacher faculty of, Department of forestry, school of life sciences, DBRA University, Agra for providing guidelines and necessary facilities during the course of study.

\section{References}

Aksamit, S.E. and Irving, F.D. 1984. Prescribed burning for lowland black spruce regeneration in Northern Minnesota. Canadian Journal of Forest Research. 14: 107-113.

Augspurger, C. K. 1984: Pathogen mortality of tropical tree seedlings: Experimental studies of the effects of dispersal distance, seedling density and light conditions. Oecologia, 61: 211-217.

Baduni, N.P., and Sharma, CM. 2001.Population structure and community analysis on different aspects of Sal savanna forest type in outer Garhwal Himalaya. Indian Forester. 127: 1001-1011.

Bhandari, B.S., 2003. Blue pine (Pinus wallichiana) forest stands of Garhwal
Himalaya: composition, population structure and diversity. Journal of Tropical Forest Science. 15: 26-36.

Bhuyan, P., Khan, M.L. and Tripathi, RS. 2003. Tree diversity and population structure in undisturbed and humanimpacted stands of tropical wet evergreen forest in Arunachal Pradesh, Eastern Himalayas India. Biodiversity Conservation. 12: 1753-1773.

Bhuyan, P., Khan, M. L. and Tripathi, R. S. 2002. Regeneration status and population structure of Rudraksh (Elaeocarpus ganitrus Roxb.) in relation to cultural disturbances in tropical wet evergreen forest of Arunachal Pradesh. Current.Science. 83: 1391-1394.

Boring, L.R., Monk, C. D. and Swank, W. T. 1981. Early regeneration of a clear cut southern Appalachian forest. Ecology. 62: 1244-1253.

Carlton, J. T. 2002. Introduced Species in U.S. Coastal Waters. Pew Oceans Commission.

Denslow, S. 1987: Tropical tree fall gaps. Annals Review Ecology and Systematics. 17: 430-441.

Dhar, U. and Samant, S. S. 1993. Endemic diversity of Indian Himalaya I. Ranunculaceae and II. Paeoniaceae. Journal of Biogeography. 20: 659-668.

Garwood, C. 1989: Tropical soil seed banks. In: Leck, M. A., Parker, V. T. \& Simpson, R. L. (eds.): Ecology of soil seed banks. Academic Press, San Diego, CA. Pp. 149-209.

Hubbel, P. and Foster, B. 1986: Biology, chance and history and the structure of tropical rainforest communities. In: Diamond, J. \& Case, J. (eds): Community ecology. Harper and Row, New York. Pp. 314-329.

Kyereth, B., Swaine, D. and Thompson, J. 1999: Effect of light on the germination of forest trees in Ghana. 
Journal of Ecology.83: 772-783.

Maram, K. M. and Khan, M.L. 1998.Regeneration status of trees in various categories of forests in Manipur. Journal of Hill Research. 11: $178-182$.

Pande, P.K., Negi, J. D. S. and Sharma, S. C. 2002. Plant species diversity, composition, gradient analysis and regeneration behaviour of some tree species in a moist temperate western Himalayan forest ecosystem. Indian Forester. 128: 869-886.

Parthasarathy, N. 2001.Changes in forest composition and structure in three sites of tropical evergreen forest around Sengaltheri, Western Ghats. Currernt Science. 80: 389-393.

Rao, P. B. 1988. Effects of environmental factors on germination and seedling growth in Querscus floribunda and Cupressustorulosa, tree species of central Himalaya. Annals of Botany. 61: 531-540.

Samant, S. S., Dhar, U. and Rawal, R. S. 1998b.Biodiversity status of a protected area of west Himalaya.1Askot Wildlife Sanctuary. Journal of
Sustainable Development and World Ecology. 5: 194-203.

Saxena, A. K. and Singh, J. S. 1984.Tree population structure of certain Himalayan forest associations and implications concerning their future composition. Vegetation 58: 61-69.

Sood, V. and Monik, B. 1991. Population structure and regeneration status of tree species in forests around Shimla, Himachal Pradesh. Van Vigyan. 29: 223-229.

Teketay, D. 1997a: The impact of clearing and conservation of dry Afromontane forests into arable land on the composition and density of soil seed banks. - Oecologia18: 557-573.

Whitmore, T. 1996: A review of some aspects of tropical rainforest seedling ecology with suggestions for further enquiry. In: Swaine, M. (ed.): The Ecology of Tropical Forest Tree Seedlings. Parthenon Publishing, Paris, Pp. 3-39.

Yamamoto, S. 1996: Gap regeneration of major tree species in different forest types of Japan. Vegetation. 127: 657669.

\section{How to cite this article:}

Sonam Rajput and Varshney, P.K. 2019. Diversity and Composition of Tree Species under Semi-arid Uttar Pradesh, India. Int.J.Curr.Microbiol.App.Sci. 8(06): 1024-1032. doi: https://doi.org/10.20546/ijcmas.2019.806.125 